\title{
Computer Software for Burden Distribution Control in
}

\section{Blast Furnace*}

\section{Introduction}

Burden distribution which affects gas flow in the blast furnace is extremely important for stable and economic operation of the furnace, and thus a more accurate technique than presently available is required for estimating it.

Therefore, a model for simulating burden distribution under several charging conditions has been developed. Thus model has been applied to actual blast furnace and is contributing to the economical operation of the Kobe No. 3 bellless B.F. and the Kakogawa No. 3 bell B.F.

\section{Simulation Model of Burden Distribution}

\section{Construction of the Model}

The model can estimate the stock-line profile and the radial distribution of particle sizes, the void fraction, and the gas velocity under several charging conditions. The stock-line profile is calculated in accordance with the following procedure and as shown in Fig. 1.

(1) The computation of the

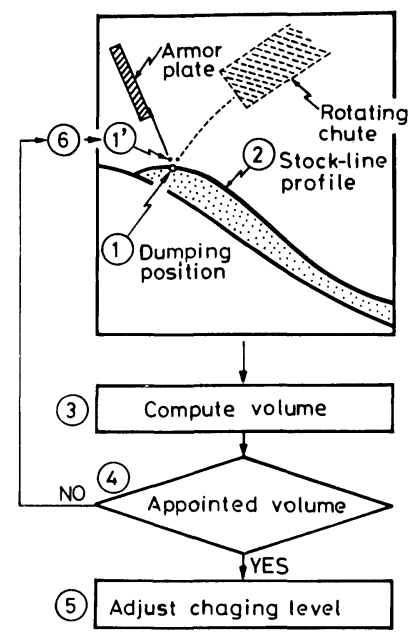

Fig. 1. Computation of stock-line profile. dumping position of the material.

(2) The repose angles of each radial position are given as functions of the dumping position, the moving distance to center, the charging volume, and the kind of burden materials.

(3) Computation of the pilling volume.

(4) Judgment as to whether the volume is equal to the appointed charging volume of the material.

(5) If the volume is equal to or greater than the appointed charging volume, the stock-line profile goes down to a given charging line.

(6) On the other hand, if the volume is smaller than the appointed charging volume, the calculation from (2) to (5) is repeated by shifting the dumping position.

The radial distributions of particle size and void fraction are given as functions of the dumping position, the moving distance to center, and the kind of burden materials.

The distribution of gas velocity in the radial direction is calculated with Ergun's equation by using the particle characteristics.

\section{Characteristics of the Model}

The model has extremely high accuracy because the following important conditions are included as variables.

1) Coke base

2) Ore/Coke

3) Pellet content in burden

4) Charging level

5) Charging sequence

6) Position of armor plate

7) Position of rotating chute

8) Particle size.

Therefore, the model is especially effective in case of changes in the production rate and the kind of burden materials.

\section{Application of the Simula- tion Model to Furnace Control}

The model has been applied to the Kobe No. 3 B.F. and the Kakogawa No. 3 B.F. As shown in Fig. 2, the estimated stock-line profile is in accordance with the observed one in the blast furnace. The model has been used effectively as a successful guide for obtaining desirable gas flow distribution.

\footnotetext{
* For further information, write to Iron \& Steel Technology Center, Kobe Steel, Ltd., 1-3-18, Wakinohama-cho, Chuo-ku, Kobe 651.

(C) 1986 ISIJ
}

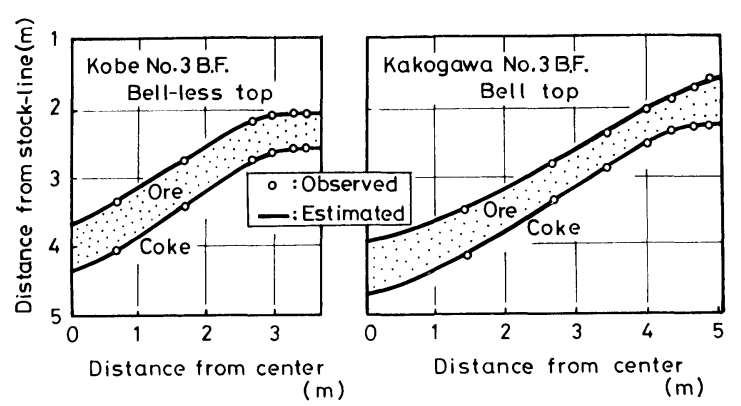

Fig. 2. Comparison of observed and estimated stock-line profiles in Kobe No. 3 B.F. and Kakogawa No. 3 B.F. 